\title{
Emotional Impact of Suicide on Active Witnesses: Predicting with Machine Learning
}

\author{
Richard Osei Agjei ${ }^{1}$, Emmanuel Awuni Kolog ${ }^{2,3, *}$, Daniel Dei ${ }^{4}$, Juliet Yayra Tengey ${ }^{5}$ \\ ${ }^{1}$ Texila America University, Department of Public Health, India \\ ${ }^{2}$ University of Ghana, Department of Operations and Management Information Systems, LG 78, Ghana \\ ${ }^{3}$ University of Eastern Finland, Department of Computer Science, School of Computing, FI-8001, Finland \\ ${ }^{4}$ Valley View University, School of Graduate Studies, Ghana \\ ${ }^{5}$ University of South Africa, Department of Psychology, South Africa
}

\section{A R T I C L E I N F O}

\section{Article history:}

Received: 12 September, 2018

Accepted: 20 October, 2018

Online: 26 October, 2018

\section{Keywords:}

Emotion

Machine Learning

Suicide

Witness-based account

\begin{abstract}
A B S T R A C T
Predicting the impact of suicide on incidental witnesses at an early stage helps to avert the possible side effect. When suicide is committed in public, incidental observers are left to grapple with it. In many cases, these incidental witnesses tend to experience the emotional side effect with time. In this study, we employed a Machine learning algorithms to predict the impact of suicide and suicidal attempt on incidental witnesses. This prediction was based on the accounts of suicide given by selected participants who have witnessed the act. The accounts, which was pre-processed into a corpus, were manually annotated with predefined emotion categories. While sadness emerged as the most salient emotional impact on the witnesses, fear was found as the lowest of the emotional impact on the witnesses. However, the machine learning prediction yielded highest in predicting depression with insignificant variations in the other emotional categories. This nonetheless shows that people who have witnessed suicide or suicidal attempts are inherently affected by some form of emotions that may require urgent attention to alleviate. By evaluating the performance of the Machine learning algorithms, the Support Vector Machine was superior, in terms its prediction, then the Multinomial Naïve Bayes algorithm. The outcome of the study contributes to the pool of research that sought to advocate the use of Machine Learning for predicting social phenomenon.
\end{abstract}

\section{Introduction}

Suicide is the termination of an individual's own life purposefully due to several factors ranging from work pressure, family pressure, peer pressure, financial pressure, academic pressure, relationship or family disharmony, social pressure, alcoholism among other known and unknown circumstances. These behaviours have become a global phenomenon where a considerable research has proven its association with cognitive and affect disorder. In recent times, the problem of suicide has become a common occurrence in both developed and under-developed countries leading to psychosocial effect and evocation of negative emotions such anger, sadness, depression, fear, worry and anxiety. Among the

*Dr. Emmanuel Awuni Kolog, Box LG 78, +233248271443 \& eakolog@ug.edu.gh global violent deaths, suicide accounts for $50 \%$ for men and $71 \%$ for women [1]. The impact of suicide on second and third parties in the society has become a social issue that requires utmost attention. Globally, the female and male ratio for suicide is 1: 4 . This ratio amounts to the seventh leading cause of death among men [2]. However, in developed countries, suicide ratio of women to men is 1:3 while low and middle-income countries have the ratio of $1: 5$.

Suicidal behaviour is any action that could cause a person to die. Before committing suicide, people exhibit suicidal behaviour that could be averted if the behaviour is detected early enough. Individual's with suicidal behaviour possess some kind of physical, psychological and economical threat to their families, friends and the society at large due to the rippling effect that 
suicide or suicidal behaviour pose. Generally, research has revealed that suicide rate is predominant in the age of 70 years and above while the second leading cause of death is among those in the age range of 15 to 29 years [1]. People commit suicide in several ways. Notable ways are Jumping from a height, drowning, going under a train, hanging, use of firearms, poisoning, selfcutting, self-immolation, and vehicular suicide, [3,4,5,6,7,8,9]. Adequate knowledge of these behaviours can inform several interventional methods and techniques.

When suicide is committed, the emotional impact of the act on witnesses is often overlooked. When the act is committed in public, incidental observers are left to grapple with it. In many cases, these incidental witnesses tend to experience the emotional side effect, which in some cases result in more severe damage. Predicting the impact of suicide of witnesses at an early stage helps to avert the possible side effect. With this in mind, we aimed at, from the point of view of using computational Machine learning techniques, tracking the emotional impact of suicide or suicidal attempt on witnesses from their accounts on witnessing suicides or attempts on suicide. The accounts were analysed with Machine learning classification techniques.

Many researchers have used machine learning applications for the detection of emotions and sentiments in text from diverse domains. In the health sector for instance, where clinical situations or assessment appear ambiguous, machine learning can be employed to validate, ascertain and strengthen clinical prediction $[10,11]$. Machine learning can be applied to clinical data and/or data on the measurable indicator of the severity of suicidal behaviour. Thus, machine learning used in conjunction with clinical data or biomarkers will help reduce the error margin in suicide risk and suicide predictions. In the arena of psychology, the use of machine learning is used predominantly to predict suicide risk among patients with mood and depression disorders [10].

\section{Theoretical Framework}

In this section, these researchers discuss related literature and theoretical foundation of emotions and their impact on suicide. Additionally, the role of using machine learning to predict suicide is discussed in this section.

\subsection{Emotion and Suicide behaviour}

Humans are a highly social species [12], and that imply that emotion prioritize response to threats and opportunities in an environment in which individuals seek to survive. When friends and family relations witness suicidal behaviours in the environment, where they live, it poses psychosocial effect and negative urgency on their emotional behaviour.

Emotion is considered to be inherently social [13] which implies that the environment and the interacting partners are key players in the evocation of positive and negative emotions. Emotions can trigger heuristic thought or swift action. It is hypothesized that emotions will bring a direct effect on quality of relationship [14], but will also moderate the relationship between its components. Thus emotions exert causal effects on the quality of our relationships [15]. Emotions represent key underpinnings for interpreting not only human decision making but also much of human behaviour as a whole. Since affective researchers have given much attention to the impact of the environment on www.astesj.com individual's emotions $[16,17,18]$, it is important to consider the impact of suicide on people who incidentally witness suicide. It is therefore crucial to understand how human emotions are affected by constant interaction with their immediate families, friends and the society as a whole [19]. The social functions and impact of society on individual's emotion have seen a considerable research, yet the general agreement among affective researchers as to whether they represent emotion is a matter of constant debate [19].

Decision serves as a conduit for both positive and negative emotion while decision making is largely influenced by emotions $[21,22,23]$. Emotion can influence suicidal thought and the depth of information which can trigger physiological, behavioural, experimental and communicational responses that enable an individual to take action quickly based on the encountered problem [23]. There is a connection between general mood and the emotional situation and happenings in an individual's environment [24]. When individual witness the incident of suicide, the emotions that are evoked are incidental emotions, which often produce influences that are unwanted and unconscious.

Suicide is a complex neurobiological phenomenon, and there is great variability in the validity of clinical assessment tools for predicting suicidal risk. Despite reaction of uncertainty surrounding witnesses' accounts of events, recent scholarship on violence has highlighted the credibility of testimonies by witnesses. Exposure to violent behaviour has the same traumatic effects on witnesses as it does on victims [25]. Like violence of all forms, witnessing suicidal behaviours affect witnesses. Extant studies have revealed that there is a high chance that witness of suicidal behaviours or suicidal attempts can develop maladaptive lifestyles [26]. In some situations, witnesses show symptoms of withdrawal in addition to maladaptibility [27]. When individuals witness suicidal behaviour the rippling effect is the retardation of the normal mental functioning of witnesses [28]. Several research techniques have been applied in determining the effect of suicide on incidental witnesses, notable is from the point of view of qualitative approach. To add is that, less studies have considered the use of predictive techniques, such as machine learning, in this domain of research. This partly informed the reason for this work, in order to ascertain if the use of machine learning can predict the emotional effect of suicide on incidental witnesses of suicide.

Considerable studies have reported that three out of every five suicide deaths yielded no clue of the suicidal communication, threats, ideation/suicide attempts or any life time history of suicidality $[29,30]$. More so, there are several illnesses that precipitate suicidal ideation among patients, and these illnesses differ from patients to patients which makes it more difficult to identify a specific biomarker(s) to predict suicide risk [31]. The ability for health professionals and providers to predict an individual's suicide risk with high certainty is limited by lack of clinical prediction rules [31]. The concept of suicidal intent/behaviour in connection with emotional dysregulation is ambiguous and contradictory while examining several studies [32, 33, 34].

Several theoretical models have been proposed in literature to provide strong theoretical and factual information that concerns the progression of puzzling behavioural and emotional outcomes. Notable of these theories of suicidal behaviour is the Interpersonal 


\section{R. Agjei et al. / Advances in Science, Technology and Engineering Systems Journal Vol. 3, No. 5, 501-509 (2018)}

Theory of Suicide [35, 36, 37]. The Interpersonal theory of suicide was earlier suggested by Joiner (2005), and later elaborated by Van-Orden et al. [36]. According to this theory, "the most dangerous form of suicidal desire is caused by the simultaneous presence of two interpersonal constructs - thwarted belongingness and perceived burdensomeness" [36: 1]. The theory further elaborates that the capability to engage in suicidal behaviour is separated from the desire to engage in suicidal behaviour. The Interpersonal Theory of suicide presents a workable construction to further improve the quality of our understanding of suicidal behaviours. This theory has proven to be one of the best lenses through which suicidal behaviour can be understood [38]. It explains the suicidal intent or ideation-death gap and provide more detail understanding of suicidal behaviour in general. The concept reveals that the interplay between perceived burdensomeness and thwarted belongingness brings about the need for an individual to die by suicide. The theory suggests that the riskiest form of suicidal desire is introduced and provoked by the interplay between perceived burdensomeness and thwarted belongingness. In addition, Christensen et al. [39] found that the interplay between perceived burdensomeness and the thwarted belongingness predicts suicidal ideation. It is interesting to note that in their findings interpersonal theory of suicide explained more variance than the epidemiological models using demographic factors, including depression, anxiety, traumatic and stressful events as predictors [39].

\subsection{Effect of Negative Emotions on Suicide}

Lerner et al. [40] view emotion as a "coordinated reactions to survival-relevant event, including cognitive and biological changes, facial and/or bodily expressions, subjective feelings, and action tendencies such as approach or withdrawal". Suicide has a close association with the influence of negative emotions. While emotion is considered as one of the factors to influence suicide, Loyo et al. [60] have reported failures in interference control, cognitive rigidity, and alterations in verbal fluidity and decisionmaking as other influences of suicide or suicidal attempts.

The emotional aspect of the Interpersonal Theory of suicide is important, in that, the three component of the theory are dangerous for any one. This danger is "significantly amplified in individuals with high level of negative urgency, as they may be more reactive to less severe environmental stressors and more highly motivated to respond to negative behaviours capable of impacting suicidal risk" [41]. Depression, anxiety and trauma comes with negative urgency. This tendency to act rashly in an attempt to reduce feelings of negative Affect: unspecified feelings; the superordinate umbrella of constructs involving emotion, mood, and emotionrelated traits [42]. Individuals with high levels of negative urgency might experience quicker, more sever increase in suicidal desire as a result of the devastating nature of their negative emotions [41]. Anestis et al. [41] argued that negative urgency does not only impact the desire for suicide, but also intensify the development of the acquired capability.

Problems associated with emotions are classified as a significant risk factor for suicide behaviour. Lees and Stimpson [43] explain that the concept of emotion, in reference to suicide, is often neglected due to the misunderstanding of emotional behaviours. Theories that bother on the state of the mind, emotional states and actions of suicidal person assimilated affect by theorising suicidality as self-harm or a form of hostility to self. Researchers have found that people participate in deadly self-harm (suicide) as a way of avoiding undesirable emotions and prevent unwanted pain. For instance, extant study suggests that being conscious of one's shortfalls leads to higher destructive emotion and for that matter, individuals cultivate a longing to end this consciousness [44]. With this effect, individuals try to lessen this consciousness and control emotion thus resulting in irrationality and worthlessness [44]. This state of reduced consciousness and rejection of emotion makes extreme actions, such as suicide, a suitable way to escape from one's self and the world.

Additionally, flashbacks, trauma, anxiety and fear of those who witnessed, experienced or experiencing suicidality is connected with problems in controlling emotions and heightened rates of suicide. Though Thomas [45] maintains that individuals with unstable emotions have inadequate knowledge of their personality or health status, thereby engage in suicidal behaviour as they recognised as the only means of coping. Research investigating emotion susceptibility to suicidal behaviour have shown that there is no single pathway or pattern to follow or track individuals who are the first time suicide attempters. This however suggests that the non-rejection of emotional reactions and poor access to professional emotional treatments could be the major means to foretell modern behaviours that are connected with suicide [46].

Anestis et al. [41] maintain that research examining the role of emotion regulation in Interpersonal Psychology Theory of Suicide has been inadequate. This is because the main focus of suicidal behaviour is lack of emotion regulation rather than behaviour. The researchers further reiterated that since emotions affect a number of psychiatric disorders with high suicide rates, it would be important to examine its relationship to suicide behaviour. Fredrickson [47] offers some theoretical and empirical support by indicating that individuals undergoing positive affect show flexible, imaginative, resourceful thought and positive emotions get rid of persistent negative emotions.

\section{Research Methodology}

This section elaborates on the methodological process of achieving the research objectives. The context of this study and the data collection procedure are as well outlined in this section. Preprocessing of the subjective responses of the participants is coded according to a pre-defined emotion category, which are presented in this section. Given the recent interest in advancing interdisciplinary research in ICT, these researchers have further analysed the collected data from the point of view of using machine learning classification techniques to predict the emotional impact of suicide on witnesses based on their accounts of suicides or suicide attempts.

\subsection{Data collection}

Data for this study was collected in Ghana. Nine hundred and seventy-eight $(\mathrm{N}=978)$ instances of emotional antecedents, regarding witness of suicides or suicidal attempts, were collected from randomly selected participants, drawing mainly from the health and other institutions of higher education. The basic rationale for the diverse sources of the data was to help understand the impact that suicide or suicide attempts have on people. The 


\section{R. Agjei et al. / Advances in Science, Technology and Engineering Systems Journal Vol. 3, No. 5, 501-509 (2018)}

collection of the data was mainly through digitally-developed questionnaires. Based on the objectives of this study, the selected participants, for this study, were tasked to respond to the questions subjectively, asking for their experience on suicide or suicidal attempts by themselves or others. The questions were purely an open-ended one, except for their demographic information. The open-ended responses from the participants were developed into a corpus that required further pre-processing for easy coding or annotation for the prediction.

\subsection{Ethical Considerations}

Given that human emotions are a sensitive phenomenon, we took into account the ethical considerations of the selected institutions and participants who responded to the questionnaires. An official letter requesting for the right to collect data was forwarded to the ministry of education and the selected institutions. The major content of the letter was to seek ethical clearance before the data was collected. This process was in compliance with the Ministry's policy of data collection.

What is more, an informed consent was obtained from each of the participants. These researchers took turn to educate the participants on the importance of this study and why their data is relevant for this study. The participants were given the opportunity to opt out at any point should they find any ethical breach or discrepancies in the process. Consequent to this, some of the participants opted out without recourse to their action. Nonetheless, the participants were assured that their data or responses will be treated confidentially and that only group findings will be reported, which will not have a direct connection to individual participants.

\subsection{Pre-processing}

These authors developed basic emotional themes (categories) that align Plutchik's eight basic emotions [59]. The development of the emotion categories was based on the manual analysis of the sample text instances. In line with Plutchik's eight basic emotions, however, we sampled $10 \%$ of the corpus and analysed to determine the common emotional themes. In the end, we deduced seven basic emotions which were found as the various emotions that the participants expressed in the sampled data. These emotion categories are worry, anger, fear, sadness, depression, surprise and no emotion. The corpus was pre-processed for the selected coders to code each instances of the corpus with the predefined emotion categories.

Three human coders with background in human psychology were selected and trained to label the instances of the corpus with the predefined emotion categories. The reason for the selection of the three coders is backed by Kolog et al. [49]. Kolog et al. [49] revealed that the emotional state of text coders influences their perception when analysing emotions in text, especially when the coders are given limited period to carry out such task. What is more, the coding process is time consuming and the outcome may not be satisfactory which could result in bad decisions on the side of the coders.

Furthermore, these researchers are of the view that people are more susceptible to the influence of their environment which has the potential to influence their perception of emotions. For example, one's domestic challenge, at a point, could influence one's decisions while analysing other people's emotional behaviour. This could, nonetheless, be attributed to the subjective nature of emotions when especially expressed in text. For this reason, the selected coders were given ample and free time to report their findings. Although, some of the instances of the data could yield or contain more than one emotion category, the coders were asked to choose the emotion category based on the most dominant emotional keywords. Below are two instances of the coded text instances of sample corpus:

\begin{abstract}
"After hearing that I felt very sad and wondered why one will take his or her life no matter what one is going through. I feel the devil has gotten it way too much into our society because I know there is a spiritual backing to suicide which is taking much control over many especially the youth" [Surprise].

"Hmmm! it very sad to hear that a fellow age mate has taken their own live. Sometimes I ask what at all they could be going through that they can share with anyone but only thought of suicide. I got depressed for couple of days before coming back to normal. Hmm only God knows Best" [Depression].
\end{abstract}

\subsection{Machine Prediction}

Having labelled the text instances by the selected coders, we further employed a machine learning technique to classify the corpus according to the pre-defined emotion categories. With this, we used WEKA machine learning software [50] for the prediction (classification) of the emotional impact of suicide on the witnesses. WEKA contains tools for data pre-processing, classification, regression, clustering, association rules, and visualization [50]. It is well-suited for developing new machine learning schemes. WEKA was developed by Machine Learning Group at the University of Waikato in the New Zealand [50]. WEKA is a collection of machine learning algorithms for data mining tasks. WEKA machine learning tool was adopted because of its versatility to analyse unstructured text. The tool is a widely known data mining tool that has generally been accepted in this research domain. Additionally, WEKA makes available source code for some of the packages, such as the classifiers, for importation into personal project. This makes it flexible to makes changes to achieve the desired outcome.

Two of the Supervised machine learning classifiers in WEKA were used in this study - Support Vector Machine (SVM) and Multinomial Naïve-Bayes (MNB) classifiers. The choice to use these classifiers is rooted in the fact that considerable studies have found these classifiers efficient and robust for text classification $[51,52]$. In WEKA package, we employed 10-folds cross validation for training and predicting of the emotions in the text. This was carried out after the corpus had been tokenised and the features extracted for the classifiers. With this in mind, the feature words were extracted as bag-of-words for the classifiers to learn and create a model for the prediction. The 10 -folds cross validation means the coded corpus was randomly partitioned into 10 equal sub-sample sizes. This suggests that the cross-validation process is repeated 10 times (the folds), with each of the 10 subsamples used exactly once as the validation data. In the subsequent paragraphs, these authors explained the various classifiers used in this study. Figure 1 illustrates the various stages in the prediction of the emotions from the witness-based accounts using supervised machine learning.

To ascertain the efficacy of the machine prediction, we evaluated the performance of the classifiers against the human 


\section{R. Agjei et al. / Advances in Science, Technology and Engineering Systems Journal Vol. 3, No. 5, 501-509 (2018)}

coded corpus. With this in mind, we computed for the recall, precision, F-measure and the accuracy of the various classifiers. While there are other evaluation measures, precision, recall and accuracy have widely been used in the evaluation of text classifiers. Recall is the proportion of the labelled instances of the gold standard that are identified and extracted by a classifier while the fraction of the automatically extracted data that was labelled correctly as the gold standard by the classifier is Precision. The Fmeasure, as referred to as the F-score, is the harmonic mean of the recall and precision. The Accuracy is the most intuitive performance measure and it is simply a ratio of correctly predicted observation to the total observations [53].

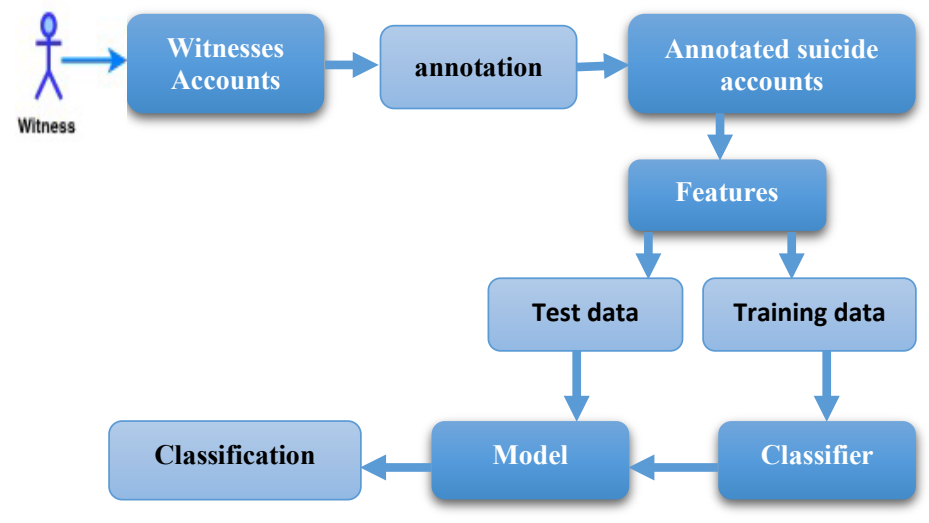

Figure 1. Conceptual classification process

\subsubsection{Support Vector Machine}

SVM is a supervised machine learning algorithm which can be used for both regression and classification. SVM is a discriminative algorithm in a sense that it is defined by constructing a hyperplane or a set of hyperplanes in high dimensional vector space. Before SVM performs classification task, feature words from the text instances is expected to be extracted through several proposed approaches such as bag-ofwords, part of speech tagging among other new advances. After constructing the hyperplane according to how the data is labelled, a model is created for predicting unseen data. Intuitively, "a good separation is achieved by the hyperplane that has the largest distance to the nearest training data point of any class (so-called functional margin), since in general, the larger the margin, the lower the generalisation error of the classifier" [51]. In this work, we used Sequential Minimal Optimisation for SVM in WEKA [50]. Research has indicated that SVM performs more accurately if the training data is very large [54]. Figure 2 shows a typical separation of data points into two categories, thus binary classification. This can, for example, be the detection and classification of input data into positive and negative polarity.

Detecting and separating the data points in a high dimensional vector space is transform by a kernel. The kernel in SVM is used to recognise patterns in the data points. So far four different kernels are available in SVM: linear kernel, polynomial kernel, Gaussian basis radial basic function (RBF) kernel and sigmoid kernel. However, in this study, all the various kernels were used to determine which of the them would yield the best performance. In the end, the linear kernel performed better, of which the results are reported in this work.

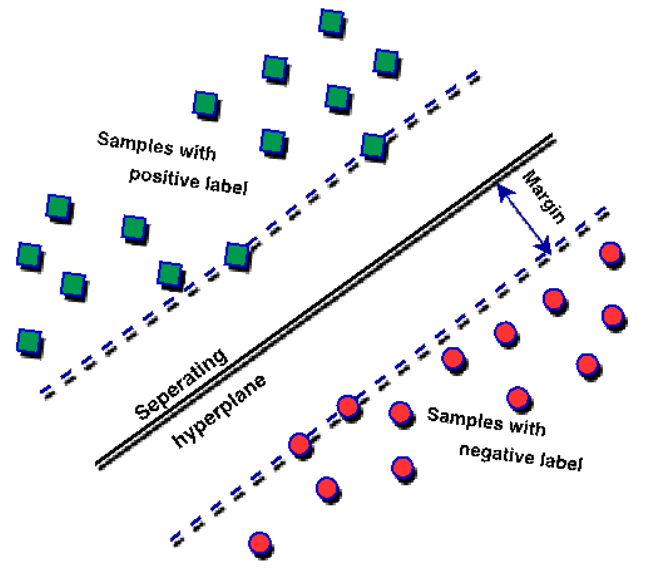

Figure 2. Maximum margin and separation of the hyperplane in SVM (Adapted from Kolog, 2017)

\subsubsection{Multinomial Nä̈ve-Bayes}

Unlike the SVM, NB is a text classifier that is based on the Bayesian theorem with strong and independence assumption in the model rather than a particular distribution of each feature. A Naive Bayes model assumes that each of the features it uses are conditionally independent of one another given some class. Naïve-Bayes is actually suited when the input data is in high dimensional vector space. What is however different is that NB can classify text instance more accurately even if the training data is small.

\section{Results and Discussion}

This section reports the findings arising from the analysis and classification of emotions in the witnesses' accounts. This considers the demographic information of the participants, and the emotional impact of the participants from the point of view of their accounts on suicidal antecedents. The results are accompanied with the discussion and backed by literature.

\subsection{Participants demography}

Of the 978 instances of the text corpus, $49 \%(n=483)$ of them were reported by male participants while the remaining $51 \%(\mathrm{n}=$ 495) were reported by the female participants. As research has proven that age is an important moderating factor in determining suicidal intent or behaviour [55], we considered the age brackets of the participants in this study. With this in mind, $4 \%(\mathrm{n}=38)$ of the participants were in the age range of 4 to $24,49 \%$ of the participants were in the range of 25 to $44,47 \%$ of them were in the range of 45 to 64 and none of the participants were above the age of 65 years. Figure 3 shows the gender and age representation of the participants in this study.

Having established the gender and age of the participants, we further prod the participants regarding the demographic information of the supposed people they have witnessed committing or attempting suicide. Although this was not the central focus of this research, the demographic information is equally vital in ascertaining the calibre of people who have committed or attempted suicide. From the responses, we deduced that $43 \%(n=419)$ were female victims of suicide as reported by the participants while the remaining $57 \%(\mathrm{n}=559)$ were male victims. This is consistent with extant findings that suicide is 


\section{R. Agjei et al. / Advances in Science, Technology and Engineering Systems Journal Vol. 3, No. 5, 501-509 (2018)}

prevalent among men than women. For example, the American foundation prevention for suicide have reported in 2018, that men die by suicide 3.53 times more than women [56]. Additionally, in 2017, WHO reported 1, 129 of suicide cases in men as against 729 suicide cases in female [57]. In Ghana, WHO have reported that 1,500 Ghanaians commit suicide every year where men record the highest number of suicides [57]. However, female has the highest number of attempted suicides and broken hearts in the various psychiatric hospitals in Ghana [57]. Conversely, the participants reported higher number of victims of suicide or suicidal attempts in men than women as in all the age groups.

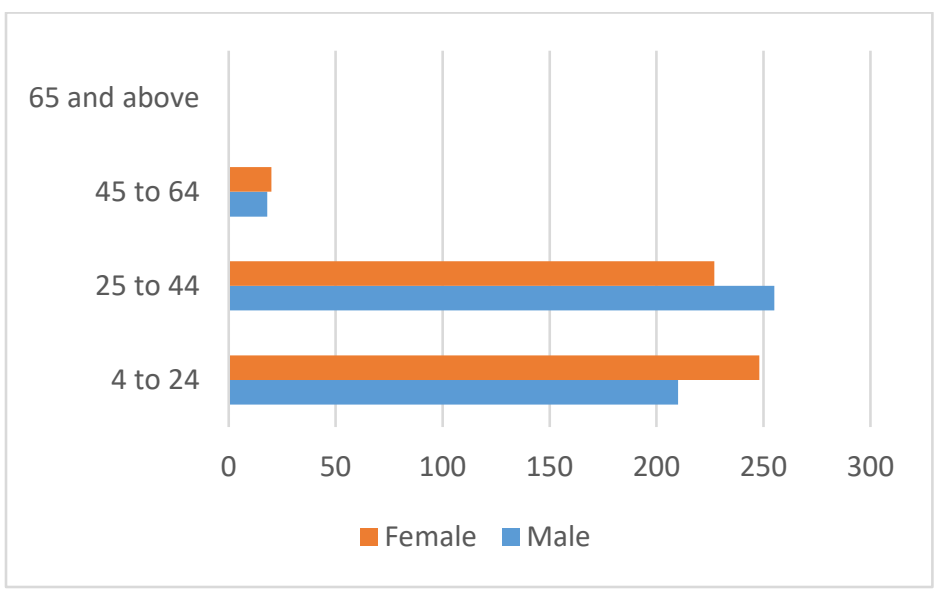

Figure 3. Gender and age representations of the participants in this study

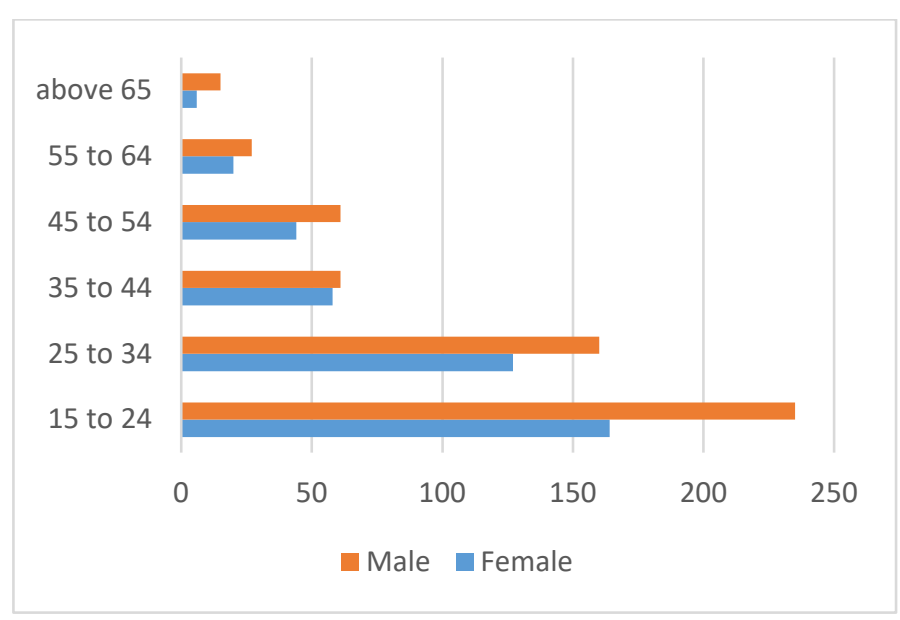

Figure 4. Age and gender representation of suicide or attempted victims by reported by the participants

Further, we collected data on the age range of the victims of suicide as reported by the participants. With this data, Figure 4 represents the age ranges of the suicide victims reported by the participants in this study. In the figure, it can be deduced that suicide is prevalent in the age of 15 to 24 years. In line with these findings, WHO in 2015 reported suicide to be prevalent among people in the age group of 15 to 29 [57]. Osafo et al. [58] found, in Ghana, that suicide behaviour is motivated by social taunting, hopelessness, and partner's infidelity. The American foundation for suicide prevention has, in real time, rated suicide as the $10^{\text {th }}$ recorded deaths in the United State of America, while in Ghana suicide is the second highest deaths [57]. The difference in suicide deaths with respect to these countries could be attributed to the diverse characteristics of each context where culture, social, economic and political colouring accounts for the variations.

\subsection{Emotional impact of suicide on witnesses}

In this section, we present the findings arising from the intercoders' agreements of emotions in the corpus. In addition, these researchers report the findings from the machine learning prediction. The inter-coder agreement kappa score was computed from all the three coders. As indicated in Table 1, a kappa score of $0.75 \mathbf{( 7 5 \% )}$ ) was obtained, which therefore indicates the variations in the coding patterns among the coders. The results from the coding is illustrated in Figure 5 and this is based on the predefined emotion categories where 1-worry, 2- anger, 3-fear, 4-sadness, 5 -depression, 6 - surprise and 7- no emotion categories. After obtaining the kappa score, the disagreements of the various instances of the data were subjected to further review and scrutiny. The deliberations among these researchers and the coders were meticulously carried out. In the end, we managed to agree on the instances of the data that were disagreed among the coders during the initial coding. The modified inter-coders' agreement of the emotions is shown in Table 1 while the initial coding by the coders is illustrated in Figure 5. The modified coded data was used as a gold standard for comparing with the machine learning prediction, as earlier established.

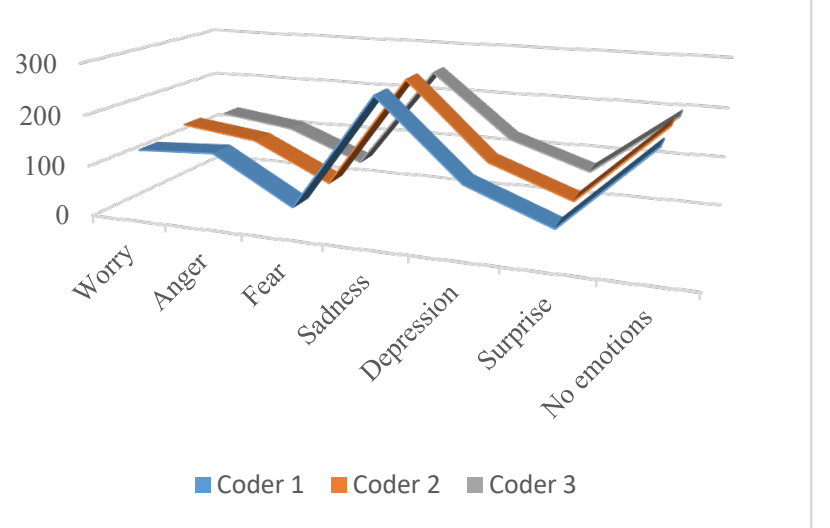

Figure 5. Coding patterns of the participants according to the emotion categories

Table 1: Inter-coded instance of emotions in the corpus

\begin{tabular}{r|l|r}
\hline$\#$ & Emotion & \# of coded instance \\
\hline 1 & Worry & $120(12 \%)$ \\
2 & Anger & $138(14 \%)$ \\
3 & Fear & $61(6 \%)$ \\
$\mathbf{4}$ & Sadness & $\mathbf{2 5 1}(\mathbf{2 6 \% )}$ \\
5 & Depression & $131(13 \%)$ \\
6 & Surprise & $70(7 \%)$ \\
7 & No emotion & $\mathbf{2 0 6 ( 2 1 \% )}$ \\
\hline & TOTAL & $\mathbf{9 7 8 ( 1 0 0 \% )}$ \\
\hline
\end{tabular}

Young et al. [61] deduced that grief, sadness, and disbelief typical of all grief, overwhelming guilt, confusion, rejection, shame, and anger are also often prominent in suicide. From Table 1, the highest coded emotion is sadness (26\% of the 978 instances). In each case of the coding, sadness appeared as the dominant emotional impact on the participants (witnesses). This is followed by no emotion category. The implication is that the majority of the witnesses felt sad after they had witnessed suicide or suicide attempts. However, the no emotion category, which appeared as the second highest, implies that the witnesses did not feel any 


\section{R. Agjei et al. / Advances in Science, Technology and Engineering Systems Journal Vol. 3, No. 5, 501-509 (2018)}

emotional impact after witnessing suicide and suicide attempts. This could also mean that the participants declined to share their emotional antecedents as requested during the data collection. The no emotion indicates $21 \%$ of the entire text instances. This implies that of the 978 participants who accounted for witnessing suicide and suicide attempts, 206 of them did not express any emotions.

From Table 1, the lowest of the emotion categories is fear. Fear accounted for $6 \%$ in the participants' responses regarding the expression of emotions. The implication of this is that only 61 out of the 978 of the participants expressed fear after they had witnessed suicides or suicide attempts. The fear emotion category may have been aroused on the grounds that the participants assumed to have been those committing or attempting the suicide. This situation is likely to trigger fear, and could eventually lead to another form of emotion category, such as depression.

\subsection{Classifiers performance}

Having predicted the emotional impact of suicide from the witnesses' accounts, there was the need to ascertain the performance of the various classifiers used in this work. Table 2 shows the Precision, Recall and F-measure of the various classifiers as elaborated in the earlier section. From the table, the performances of the machine learning classifiers for each of the emotion categories are reported, including the overall performance of the classifiers. Aside the no emotion category, the highest predicted emotion by the SVM classifier is depression. To add is that, the SVM classifier yielded $90 \%$ accuracy for predicting depression. With this accuracy, the SVM produced $92 \%$ Recall and $87 \%$ Precision from predicting depression in the text corpus. However, worry was the lowest of the emotion categories which was predicted by the SVM classifier though this exceeded the general acceptable threshold of $70 \%$. This implies that, of the 131 instances that expressed depression, 92\% of them were identified and tagged, however only $87 \%$ of the identified proportion of the instances were accurately tagged and matched with the gold standard. This score is higher than the threshold of the inter-coder agreement of the $75 \%$ reported earlier.

In the same vein, the highest individual emotion category predicted by the MNB classifier is depression. From Table 2, the MNB classifier performed satisfactorily as the Recall, Precision and the F-measure yielded $88 \%, 90 \%$ and $89 \%$ respectively. These scores are beyond the generally acceptable $70 \%$ threshold. The implication is that while $88 \%$ of the 131 instances of the data was identified and recognised by the classifier, $90 \%$ was accurately predicted as the gold standard instance of the corpus. Just like the SVM, worry was found to be the lowest in terms of predicting emotions in the text.

In the nutshell, the best performing classifier is the SVM (accuracy $=78 \%$ ), of which the Precision, Recall and F-measure exceeded the acceptable threshold of $70 \%$. Although the difference in the performance is not significant, both classifiers can be concluded as suitable for predicting emotions in the witnesses' accounts with regards to suicide or suicide attempts.

Although the two classifiers had scores greater than the threshold, the SVM classifier was superior over the MNB. We conclude that machine learning, as compared with humans, can predict emotions in text that can be likened to humans.
Table 2: Evaluation results of the various machine learning predictions

\begin{tabular}{l|r|r|r|r|r|r}
\hline & \multicolumn{3}{|c|}{ SVM } & \multicolumn{3}{|c}{ MNB } \\
\hline Emotion & R & P & F & R & P & F \\
\hline Worry & 73 & 67 & 70 & 67 & 69 & 68 \\
Anger & 82 & 75 & 78 & 73 & 80 & 76 \\
Fear & 76 & 89 & 72 & 72 & 71 & 72 \\
Sadness & 78 & 72 & 75 & 68 & 65 & 67 \\
Depression & $\mathbf{9 2}$ & $\mathbf{8 7}$ & $\mathbf{9 0}$ & $\mathbf{8 8}$ & $\mathbf{9 0}$ & $\mathbf{8 9}$ \\
Surprise & 74 & 77 & 75 & 78 & 74 & 76 \\
No emotion & $\mathbf{9 3}$ & $\mathbf{9 4}$ & $\mathbf{9 4}$ & 89 & 90 & 90 \\
\hline TOTAL & $\mathbf{8 1}$ & $\mathbf{8 0}$ & $\mathbf{7 9}$ & $\mathbf{6 6}$ & $\mathbf{6 7}$ & $\mathbf{6 7}$ \\
\hline
\end{tabular}

\section{Conclusion}

We investigated the emotional impact of suicide on incidental witnesses using Machine Learning classification techniques. Usually, this domain of research is overlooked as many researchers focus on the cause and impact of suicide behaviour. To do this, we collected subjective data about some suicidal antecedents of people from a random setting. The collected data was pre-processed into corpus for suitability and easy annotation with predefined emotion categories adopted from Plutchik. The corpus, which was coded with the predefined emotion categories, was further analysed using Machine Learning classifiers - SVM and MNB. We employed 10-folds cross validation strategy in the classification of emotions in the corpus.

With regard to the manual analysis of the emotions in the corpus by well-trained coders, sadness emerged as the most salient emotion expressed by the participants (witnesses) while fear was found to be the lowest emotion category expressed by the participants (witnesses). However, the machine learning prediction yielded highest in predicting depression with insignificant variations in the other emotional categories. This nonetheless shows that people who have witnessed suicide or suicidal attempts are inherently affected by some form of emotions that may require urgent attention to alleviate. In addition, through the witnesses' accounts, the study revealed that suicide and suicidal attempts occur in the age of 15 to 24 , with men being the highest victim.

By evaluating the performance of the classifiers, the SVM classifier emerged as superior in terms of predicting the emotional impact of suicide on the witnesses (participants). By comparing the performance of the various classifiers with humans, we conclude that the Machine Learning algorithms can predict emotions in text as humans. The outcome of the study contributes to the pool of research that sought to advocate for the use of machine learning for predicting social phenomenon, especially in text, for complementing decisions. Additionally, the finding has demonstrated the emotional impact of suicide on incidental witnesses.

\section{Conflict of Interest}

The authors declare no conflict of interest.

\section{References}

[1] World Health Organization. World health statistics 2015. World Health Organization, 2015.

[2] UNICEF. Study of prevalence and dynamics of suicide among children and young people (12-24 years of age) in SUGHD REGION, TAJIKISTAN. 2013, pp. 1-88. 


\section{R. Agjei et al. / Advances in Science, Technology and Engineering Systems Journal Vol. 3, No. 5, 501-509 (2018)}

[3] R.M. Berman, A. Cappiello, A. Anand, D.A. Oren, G.R. Heninger, D.S. Charney \& J.H. Krystal. "Antidepressant effects of ketamine in depressed patients." Biol Psychiatry. 47(4):351-4, 2000.

[4] W. Eisele, D.T. Reay \& A. Cook. "Sites of Suicidal Gunshot Wounds", J Forensic Science, 26 (3), 1981.

[5] D. Klonsky \& M.M Alexis. "The Three-Step Theory (3ST): A New Theory of Suicide Rooted in the Ideation-to-Action. Framework". International Journal of Cognitive Therapy, 8(2), 114-129, 2015.

[6] HKJC Centre for Suicide Research and Prevention, University of Hong Kong. Method Used in Completed Suicide, 2006. Available at http://csrp.hku.hk/WEB/eng/statistics.asp\#3.

[7] J.J. Card. "Lethality of suicidal methods and suicide risk: Two distinct concepts", Omega 5, 1974

[8] V. Admiraal,. "Guide to a Humane Self-Chosen Death Alt Suicide Holiday". Accessed on $24^{\text {th }}$ April 2018 on http://ash2.wikkii.com/wiki/Helium, with their information taken from a range of sources but including, 2006

[9] P. Nitschke, \& F. Stewart. "The Peaceful Pill eHandbook", 2009.

[10] P. Passos \& J.Y Chow, "Interpersonal coordination in team sports," in Interpersonal Coordination and Performance in Social Systems, ed. J. Y. Chow (New York, NY: Routledge), 154-164, 2016.

[11] M.J. Just, L. Pan, V. L. Cherkassky, D.L. McMakin, C. Cha., M.K. Nock \& D. Brent. "Machine learning of neural representations of suicide and emotion concepts identifies suicidal youth". Nature Human Behavior, 2017. October 30. doi:10.1038/s41562-017-0234-y

[12] E.O. Wilson. "The Meaning of Human Existence". $1^{\mathrm{ST}}$ ed. W. W. Norton \& Company, 2014, 192pages.

[13] G.A. Van-Kleef, A. Cheshin, A. H. Fischer \& I.K Schneider.. "Editorial: The social nature of emotions". Frontiers in Psychology, 7(896), 1-5, 2016.

[14] D. Keltner \& J. Haidt. "Social functions of emotions at four levels of analysis". Cognition and Emotion 13: 505-21, 1999.

[15] P.M. Niedenthal \& M. Brauer. "Social Functionality of Human Emotion" Annual Review of Psychology, Vol. 63, pp. 259-285, 2012.

[16] A.S. Morris, J.S. Silk, L. Steinberg, S.S. Myers \& L.R. Robinson. "The Role of the Family Context in the Development of Emotion Regulation". Social Development. Author manuscript; Published in final edited form as: Soc Dev. 16(2): 361-38, 2007.

[17] D. Keltner \& J.S Lerner. "Emotion." In The handbook of social psychology, ed. DT Gilbert, ST Fiske, G Lindzey, pp. 317-52. New York, NY: Wiley, 2010.

[18] P. Ekman. "Emotions revealed: Recognizing faces and feelings to improve communication and emotional life". New York, NY: Holt, 2007.

[19] E.A. Kolog, C.S. Montero \& M. Tukiainen. "Development and Evaluation of automated e-counselling system for emotion and sentiment analysis". The electronic journal of information systems evaluation. 21(1), 1-19, 2018.

[20] D. Keltner, K. Oatley, J.M. Jenkins. "Understanding emotions". Hoboken, NJ: Wiley, 2004

[21] R. Coughlan \& T. Connolly. "Predicting affective responses to unexpected outcomes." Organizational Behavior and Human Decision Processes, 85(2), 211-225, 2001.

[22] B.A. Mellers, "Choice and the relative pleasure of consequences". Psychological Bulletin 126: 910-24, 2001.

[23] N.H. Frijda \& B. Mesquita. "The social roles and functions of emotions". In S. Kitayama \& H. R. Markus (Eds.), Emotion and culture: Empirical studies of mutual influence. Washington, DC: American Psychological Association, 1994.

[24] B. Parkinson. "Emotions are social”. Br. J. Psychol. 1996, 87, 663-683. doi: 10.1111/j.2044-8295.1996.tb02615.x

[25] N.E. Dowd, D.G. Singer \& R.F. Wilson, eds. "Handbook of Children, Culture, and Violence". California: Sage, 2006.

[26] D.A.Brent, J. Perper, G. Moritz, A. Friend, J. Schweers, C. Allman, L. McQuiston,M.B. Boylan, C. Roth, \& L. Balach. "Adolescent witnesses to a peer suicide". Journal of American Academy of Child and Adolescent Psychiatry, 32(6): 1184-1188, 1993.

[27] I.T. Young, A. Iglewicz, D. Glorioso, N. Lanouette, K. Seay, M. Ilapakurti, \& S. Zisook. Suicide Bereavement and Complicated Grief. Dialogues in Clinical Neuroscience, 14(2): 177-186, 2012.

[28] P. Goldblum, D. L. Espelage, J. Chu, \& B. Bongar eds.. "Youth Suicide and Bullying: Challenges and Strategies for Prevention and Intervention". Oxford: Oxford University Press. 2015.

[29] F.B. Ribeiro, E.A.T. Lanna, M.A.D Bomfim, J.L. Donzele, M. Quadros, P. Cunha \& S.L de. "True and apparent digestibility of protein and amino acids of feed in Nile tilapia". Rev. Bras. Zootec., 40 (5): 939-946, 2011

[30] K. Posner, G.K. Brown, B. Stanley, D.A. Brent, K.V. Yershova, M.A. Oquendo, G.W. Currier, G.A. Melvin, L. Greenhill, S. Shen \& J.J. Mann. "The Columbia-Suicide Severity Rating Scale: initial validity and internal consistency findings from three multisite studies with adolescents and adults”. Am J Psychiatry, 168(12):1266-77, 2011.
[31] M.A. Oquendo, G.M. Sullivan, S. Katherin , E. Baca-Garcia, B.H. Stanley, M.E. Sublette \& J.J. Mann. "Toward a Biosignature for Suicide". The American Journal of Psychaitry, 171 (12,), pp. 1259-1277, 2014.

[32] E.A. Selby, M.A. Anestis, W. Theodore \& E. J. Thomas E. J. “An Exploration of the Emotional Cascade Model in Borderline Personality Disorder" J Abnorm Psychol., 118(2): 375-38, 2009.

[33] N. Garnefski, V. Kraaij, \& P. Spinhoven. Negative Life Events Cognitive Emotional Regulation and Emotional Problems. Personality and Individual Differences, 30, 1311-1327, 2001.

[34] R.L. Tamas, D. Menkes \& R.S. El-Mallakh. S”timulating research: a prospective, randomized, double-blind, sham-controlled study of slow transcranial magnetic stimulation in depressed bipolar patients." Journal of Neuropsychiatry Clin Neurosci;19:198-199, 2007.

[35] T. Joiner. "Why people die by suicide". Cambridge, MA, US: Harvard University Press; 2005

[36] K.A. Van Orden, T.K. Witte, K.C. Cukrowicz, S.R. Braithwaite, E.A. Selby \& T.E. Joiner Jr. "The interpersonal theory of suicide". Psychological review, 117(2), 575, 2010.

[37] C. Zlotnick, T.M. Shea, K. Rosen, E. Simpson, K. Mulrenin, A. Begin, et al. "An affect-management group for women with posttraumatic stress disorder and histories of childhood sexual abuse". Journal of Traumatic Stress, 10, pp. 425-436, 1997

[38] T.J. Lynch, D.W. Bell \& R. Sordella. "Activating mutations in the epidermal growth factor receptor underlying responsiveness of non-small-cell lung cancer to gefitinib". Engl Journal of Med.; 350: 2129-2139, 2004.

[39] J.O. Christensen \& S. Knardahl. "Work and headache: a prospective study of psychological, social, and mechanical predictors of headache severity". Pain, 153(10):2119-2132, 2012.

[40] I. Lerner, B.C. Armstrong, \& R. Frost. "What can we learn from learning models about sensitivity to letter-order in visual word recognition?" Journal of Memory and Language, 77, 40-58, 2014.

[41] M.D. Anestis, C.L. Bagge, M.T. Tull, \& T.E. Joiner. "Clarifying the role of Emotion deregulation in the interpersonal-psychological theory of suicidal behaviour in an undergraduate sample". Journal of Psychiatric Research, 45, 603-611, 2011

[42] S.P. Whiteside \& D.R. Lynam. "The Five Factor Model and impulsivity: using a structural model of personality to understand impulsivity." Personality and Individual Differences,, 30(4), 669-689. 2001. doi: 10.1016/S01918869(00)00064-7

[43] M.D. Anestis \& T.E. Joiner. "Examining the role of emotion in suicidality: Negative urgency as an amplifier of the relationship between components of the interpersonal-psychological theory of suicidal behavior and lifetime number of suicide attempts". Journal of affective disorders, 129(1), 261-269, 2011.

[44] Baumeister, R. F. "Suicide as escape from self”. Psychological Review, 97(1), 90-113, 1990.

[45] A.L. Thomas. "Suicidal Thoughts and Behaviour among Black College Students: Examining the Impact of Distress Tolerance and Social Support on Suicidality.” Masters Theses \& Specialist Projects, 2015.

[46] K. Rajappa, M. Gallagher, \& R. Miranda. "Emotion dysregulation and vulnerability to suicidal ideation and attempts." Cognitive Therapy Res, 2012 36-833-839. DOI:10.1007/s10608-011-9419-2

[47] B.L Fredrickson. "The role of positive emotions in positive psychology: The broaden-and-build theory of positive emotions". American Psychologist, 56, 218-226. 2011

[48] E.A. Kolog, C. Suero Montero, \& e. Sutinen. "Annotation agreement of emotions in text: The influence of counsellors emotional state on their emotion perception". In proceedings of international conference on advance learning technologies 2016

[49] E.A. Kolog, \& C. Suero Montero. "Towards automated e-counselling system based on counsellors' emotion perception". Education and information technologies, pp. 1-23, 2018

[50] M. Hall, E. Frank., G. Holmes, B. Pfahringer, P. Reutemann, \& I.H. Witten, "The WEKA Data Mining Software: An Update. SIGKDD Explorations", 2009

[51] E.A. Kolog, C.S. Montero \& T. Toivonen. "Using machine learning for sentiment and social influence analysis in text". In: Rocha, A., Guarda, T. (eds.) Proceedings of the International Conference on Information Technology \& Systems (ICITS 2018). AISC, vol. 721, pp. 453-463. Springer, Cham (2018)

[52] A. Tripathy, A. Anand \& S.K. Rath. "Document-level sentiment classification using hybrid machine learning approach". Knowledge and Information Systems. 2017; 53(3):805-31.

[53] Cohen, J. "Coefficient of agreement for nominal scales." Educational and Psychological Measurement, 20(1), 37-46, 1960.

[54] E. A. Kolog. Detecting Emotions in Students' Generated Content: An Evaluation of EmoTect System. In International Conference on Technology in Education, 2018, pp. 235-248. Springer, Singapore, 2018. 
[55] N. Ibrahim, N. Amit, N.C. Din \& H.C. Ong. Gender differences and psychological factors associated with suicidal ideation among youth in Malaysia. Psychology research and behaviour management, 10, 129, 2017.

[56] American foundation for suicide prevention (AFSP). "Suicide statistics". Available on <https://afsp.org/about-suicide/suicide-statistics/> Accessed on the $13^{\text {th }}$ April 2018.

[57] World Health Organization. "World health statistics 2016: monitoring health for the SDGs sustainable development goals". World Health Organization, 2016.

[58] J. Osafo, C.S. Akotia, J. Andoh-Arthur \& E.N.B. Quarshie, "Attempted suicide in Ghana: Motivation, stigma, and coping." Death studies, 39(5), 274280. 2015.

[59] R. Plutchik. Emotion: Theory, research, and experience: Theories of emotion. New York: Academic, 1, 399, 1980.

[60] L. Loyo, E. Martínez-Velázquez \& J. Ramos-Loyo. "Influence of emotions on executive functions in suicide attempters." Suicidal Online, 4(1), 42-55, 2013. 